\title{
ASYMPTOTIC BEHAVIOR OF SOLUTIONS OF POINCARÉ DIFFERENCE EQUATIONS
}

\author{
WILLIAM F. TRENCH
}

(Communicated by Charles Pugh)

ABSTRACT. It is shown that if the zeros $\lambda_{1}, \lambda_{2}, \ldots, \lambda_{n}$ of the polynomial

$$
q(\lambda)=\lambda^{n}+a_{1} \lambda^{n-1}+\cdots+a_{n}
$$

are distinct and $r$ is an integer in $\{1,2, \ldots, n\}$ such that $\left|\lambda_{s}\right| \neq\left|\lambda_{r}\right|$ if $s \neq r$, then the Poincare difference equation

$$
y(n+m)+\left(a_{1}+p_{1}(m)\right) y(n+m-1)+\cdots+\left(a_{n}+p_{n}(m)\right) y(m)=0
$$

has a solution $y_{r}$ such that (A) $y_{r}(m)=\lambda_{r}^{m}(1+o(1))$ as $m \rightarrow \infty$, provided that the sums $\sum_{j=m}^{\infty} p_{i}(j) \quad(1 \leq i \leq n)$ converge sufficiently rapidly. Our results improve over previous results in that these series may converge conditionally, and we give sharper estimates of the $o(1)$ terms in (A).

\section{INTRODUCTION}

We consider the Poincaré difference equation

$$
y(n+m)+\left(a_{1}+p_{1}(m)\right) y(n+m-1)+\cdots+\left(a_{n}+p_{n}(m)\right) y(m)=0,
$$

where $a_{n} \neq 0$, the polynomial

$$
q(\lambda)=\lambda^{n}+a_{1} \lambda^{n-1}+\cdots+a_{n}
$$

has distinct zeros $\lambda_{1}, \lambda_{2}, \ldots, \lambda_{n}$, and

$$
\lim _{m \rightarrow \infty} p_{k}(m)=0, \quad 1 \leq k \leq n \text {. }
$$

Under these assumptions it is natural to ask whether (1) has solutions $y_{1}$, $y_{2}, \ldots, y_{n}$ which behave asymptotically in some sense like the solutions $x_{r}(m)=\lambda_{r}^{m} \quad(1 \leq r \leq n)$ of the constant coefficient equation

$$
x(n+m)+a_{1} x(n+m-1)+\cdots+a_{n} x(m)=0 .
$$

If $\lambda_{1}, \lambda_{2}, \ldots, \lambda_{n}$ have distinct moduli, then Poincaré's theorem [5] asserts that every nontrivial solution of (1) exhibits the asymptotic behavior

$$
\lim _{m \rightarrow \infty} \frac{y(m+1)}{y(m)}=\lambda_{r}
$$

Received by the editors March 27, 1991; presented to the Society January 9, 1992.

1991 Mathematics Subject Classification. Primary 39A10.

Key words and phrases. Poincaré difference equation, asymptotic behavior, Perron's theorem, conditional convergence. 
for some $r$ in $\{1,2, \ldots, n\}$ and Perron's theorem [4] asserts that (1) has solutions $y_{1}, y_{2}, \ldots, y_{n}$ such that

$$
\lim _{m \rightarrow \infty} \frac{y_{r}(m+1)}{y_{r}(m)}=\lambda_{r}, \quad 1 \leq r \leq n .
$$

The conclusions of Poincaré's and Perron's theorems are weak, since (3) does not imply that $y_{r}(m)-\lambda_{r}^{m}$ becomes small (i.e., $o\left(\lambda_{r}^{m}\right)$ ) as $m \rightarrow \infty$. (We will use $O$ and $O$ in the usual way to indicate asymptotic behavior as $m \rightarrow \infty$.) To obtain this conclusion it is necessary to replace (2) with a stronger condition. For example, the following theorem is due to Evgrafov [2].

Theorem 1. Suppose that the zeros $\lambda_{1}, \lambda_{2}, \ldots, \lambda_{n}$ of $q(\lambda)$ are distinct and

$$
\sum_{m=0}^{\infty}\left|p_{k}(m)\right|<\infty, \quad 1 \leq k \leq n .
$$

Then (1) has solutions $y_{1}, \ldots, y_{n}$ such that

$$
y_{r}(m)=\lambda_{r}^{m}(1+o(1)), \quad 1 \leq r \leq n .
$$

The following theorem of Gelfond and Kubenskaya [3] provides an estimate of the $o(1)$ term in (4).

Theorem 2. Suppose that $\left|\lambda_{1}\right|<\left|\lambda_{2}\right|<\cdots<\left|\lambda_{n}\right|$ and there is a nonincreasing sequence $\beta$ such that

$$
\begin{gathered}
\left|p_{i}(m)\right| \leq \beta(m), \quad m=0,1, \ldots, 1 \leq i \leq n, \\
\lim _{m \rightarrow \infty} \frac{\beta(m+1)}{\beta(m)}=1,
\end{gathered}
$$

and $\sum^{\infty} \beta(m)<\infty$. Let $\gamma(m)=\sum_{j=m}^{\infty} \beta(j)$. Then (1) has solutions $y_{1}, \ldots$, $y_{n}$ such that

$$
y_{r}(m)=\lambda_{r}^{m}(1+O(\gamma(m))), \quad 1 \leq r \leq n .
$$

Coffman [1] has shown that (5) can be weakened to

$$
\liminf _{m \rightarrow \infty} \frac{\beta(m+1)}{\beta(m)}>\max _{1 \leq i<n}\left(\frac{\left|\lambda_{i}\right|}{\left|\lambda_{i+1}\right|}\right) \text {. }
$$

Theorems 1 and 2 do not apply if any of the series $\sum_{m=0}^{\infty} p_{i}(m) \quad(1 \leq i \leq n)$ converge conditionally. Moreover, even if these series converge absolutely, the estimate of the order of convergence in (6) may be too conservative, as our examples in $\S 3$ will illustrate.

The following theorem is our main result.

Theorem 3. Suppose that the zeros $\lambda_{1}, \lambda_{2}, \ldots, \lambda_{n}$ of $q(\lambda)$ are distinct, and let $0<\left|\lambda_{1}\right| \leq\left|\lambda_{2}\right| \leq \cdots \leq\left|\lambda_{n}\right|$. Suppose also that the series $\sum_{m=0}^{\infty} p_{i}(m) \quad(1 \leq i \leq n)$ converge (perhaps conditionally) and there are nonincreasing sequences $\phi$ and $\psi$ such that $\lim _{m \rightarrow \infty} \phi(m)=\lim _{m \rightarrow \infty} \psi(m)=0, \psi(m)=o(\phi(m))$,

$$
\sum_{j=m}^{\infty} p_{i}(j)=O(\phi(m)), \quad 1 \leq i \leq n,
$$


and

$$
\sum_{j=m}^{\infty}\left|p_{i}(j)\right| \phi(n+j-i)=O(\psi(m)), \quad 1 \leq i \leq n .
$$

Let $r$ be an integer in $\{1,2, \ldots, n\}$ such that $\left|\lambda_{r}\right| \neq\left|\lambda_{s}\right|$ if $r \neq s$. If $r>1$, suppose also that there is an integer $M_{1}$ and a number $\rho$ such that

$$
1<\rho<\left|\lambda_{r} / \lambda_{r-1}\right|
$$

and $\rho^{m} \phi(m)$ and $\rho^{m} \psi(m)$ are nondecreasing for $m \geq M_{1}$. Then (1) has a solution $y_{r}$ such that

$$
y_{r}(m)=\lambda_{r}^{m}(1+O(\phi(m))), \quad m \rightarrow \infty .
$$

The assumption concerning $\rho$ is equivalent to the conditions

$$
\liminf _{m \rightarrow \infty} \frac{\phi(m+1)}{\phi(m)}>\left|\frac{\lambda_{r-1}}{\lambda_{r}}\right| \text { and } \liminf _{m \rightarrow \infty} \frac{\psi(m+1)}{\psi(m)}>\left|\frac{\lambda_{r-1}}{\lambda_{r}}\right|,
$$

which is related to Coffman's condition (7).

\section{Proof of Theorem 3}

We will prove Theorem 3 by means of a series of lemmas. For convenience we rewrite (1) as

$$
y(n+m)+a_{1} y(n+m-1)+\cdots+a_{n} y(m)=-\mathscr{L} y(m),
$$

where

$$
\mathscr{L} y(m)=p_{1}(m) y(n+m-1)+p_{2}(m) y(n+m-2)+\cdots+p_{n}(m) y(m) .
$$

By variation of parameters, $y$ is a solution of (1) if and only if

$$
y(m)=\sum_{k=1}^{n} \lambda_{k}^{m} u_{k}(m)
$$

where

$$
\sum_{k=1}^{n} \lambda_{k}^{m+i} \Delta u_{k}(m)=-\delta_{\text {in }} \mathscr{L} y(m), \quad 1 \leq i \leq n .
$$

Solving this system yields

$$
\Delta u_{k}(m)=-A_{k} \lambda_{k}^{-m} \mathscr{L} y(m) \text { with } A_{k}=1 / \lambda_{k} q^{\prime}\left(\lambda_{k}\right) .
$$

Now let $M$ be a positive integer which we will specify further below. For now we assume that if $r>1$ then $\rho^{m} \phi(m)$ and $\rho^{m} \psi(m)$ are nondecreasing for $m \geq M$ for some $\rho$ satisfying (10). From (12) and (13), if the sequence $y_{r}$ satisfies

$$
\begin{aligned}
y_{r}(m)= & \lambda_{r}^{m}-\sum_{k=1}^{r-1} A_{k} \sum_{j=M}^{m-1} \lambda_{k}^{m-j} \mathscr{L} y_{r}(j) \\
& +\sum_{k=r}^{n} A_{k} \sum_{j=m}^{\infty} \lambda_{k}^{m-j} \mathscr{L} y_{r}(j), \quad m \geq M
\end{aligned}
$$

(where the first sum is vacuous if $r=1$ ), then $y_{r}$ satisfies (1) for $m \geq M$. 
It is convenient to rewrite (14) in terms of the sequence $v_{r}$ defined by

$$
v_{r}(m)=\lambda_{r}^{-m} y_{r}(m)-1 \text {. }
$$

Then

$$
\mathscr{L} y_{r}(j)=P_{r}(j)+\mathscr{M}_{r} v_{r}(j)
$$

where

$$
P_{r}(j)=\sum_{i=1}^{n} \lambda_{r}^{n+j-i} p_{i}(j)
$$

and

$$
\mathscr{M}_{r} u(j)=\sum_{i=1}^{n} \lambda_{r}^{n+j-i} p_{i}(j) u(n+j-i)
$$

for any sequence $u$. From (14)-(16),

$$
\begin{aligned}
v_{r}(m)= & F_{r}(m)-\sum_{k=1}^{r-1} A_{k}\left(\frac{\lambda_{k}}{\lambda_{r}}\right)^{m} \sum_{j=M}^{m-1} \lambda_{k}^{-j} \mathscr{M}_{r} v_{r}(j) \\
& +\sum_{k=r}^{n} A_{k}\left(\frac{\lambda_{k}}{\lambda_{r}}\right)^{m} \sum_{j=m}^{\infty} \lambda_{k}^{-j} \mathscr{M}_{r} v_{r}(j),
\end{aligned}
$$

where

(20) $\quad F_{r}(m)=-\sum_{k=1}^{r-1} A_{k}\left(\frac{\lambda_{k}}{\lambda_{r}}\right)^{m} \sum_{j=M}^{m-1} \lambda_{k}^{-j} P_{r}(j)+\sum_{k=r}^{n} A_{k}\left(\frac{\lambda_{k}}{\lambda_{r}}\right)^{m} \sum_{j=m}^{\infty} \lambda_{k}^{-j} P_{r}(j)$.

If $v_{r}$ satisfies (19), then the sequence $y_{r}$ defined by

$$
y_{r}(m)=\left(1+v_{r}(m)\right) \lambda_{r}^{m}
$$

satisfies (1). This motivates us to look for $v_{r}$ as a fixed point (sequence) of the transformation

$$
v=F_{r}+\mathscr{T}_{r} u
$$

where

$$
\begin{aligned}
\mathscr{T}_{r} u(m)= & -\sum_{k=1}^{r-1} A_{k}\left(\frac{\lambda_{k}}{\lambda_{r}}\right)^{m} \sum_{j=M}^{m-1} \lambda_{k}^{-j} \mathscr{M}_{r} u(j) \\
& +\sum_{k=r}^{n} A_{k}\left(\frac{\lambda_{k}}{\lambda_{r}}\right)^{m} \sum_{j=m}^{\infty} \lambda_{k}^{-j} \mathscr{M}_{r} u(j) .
\end{aligned}
$$

Now let $M \geq M_{1}$ and let $\mathscr{B}$ be the Banach space of sequences $u=$ $\{u(m)\}_{m=M}^{\infty}$ such that $u(m)=O(\phi(m))$, with norm

$$
\|u\|=\sup _{m \geq M}\{|u(m)| / \phi(m)\} .
$$

We will show that (22) is a contraction mapping of $\mathscr{B}$ into itself if $M$ is sufficiently large. 
Lemma 1. Suppose that the series $\sum^{\infty} w(j)$ converges, and let $\sigma$ be a nonincreasing sequence such that

$$
\sigma(m) \geq \sup _{\nu \geq m}|W(\nu)|, \quad \text { where } W(m)=\sum_{j=m}^{\infty} w(j) .
$$

Let $\gamma$ be a complex constant.

(a) If $|\gamma|<1$ then

$$
\left|\sum_{j=m}^{\infty} \gamma^{j} w(j)\right| \leq K_{1}|\gamma|^{m} \sigma(m),
$$

where $K_{1}$ depends only on $\gamma$.

(b) If $|\gamma|>1$ and there is a number $\rho$ such that $1<\rho<|\gamma|$ and $\rho^{m} \sigma(m)$ is nondecreasing for $m \geq M$, then

$$
\left|\sum_{j=M}^{m-1} \gamma^{j} w(j)\right| \leq K_{2}|\gamma|^{m} \sigma(m), \quad m \geq M+1,
$$

where $K_{2}$ is a constant which depends only on $\gamma$ and $\rho$.

Proof. (a) Summation by parts yields

$\sum_{j=m}^{N} \gamma^{j} w(j)=-\gamma^{N} W(N+1)+\gamma^{m} W(m)+\sum_{j=m+1}^{N}\left(\gamma^{j}-\gamma^{j-1}\right) W(j), \quad m<N$.

Letting $N \rightarrow \infty$ and applying routine estimates yields (25) with

$$
K_{1}=1+\frac{|1-\gamma|}{1-|\gamma|}
$$

(b) Summation by parts yields

$$
\sum_{j=M}^{m-1} \gamma^{j} w(j)=\gamma^{M} W(M)-\gamma^{m-1} W(m)+\left(1-\frac{1}{\gamma}\right) \sum_{j=M+1}^{m-1} \gamma^{j} W(j) ;
$$

therefore,

$$
\begin{aligned}
\left|\gamma^{-m} \sum_{j=M}^{m-1} \gamma^{j} w(j)\right| \leq & \frac{|\gamma|^{M-m}}{\rho^{M}}\left(\rho^{M} \sigma(M)\right)+\frac{1}{|\gamma|} \sigma(m) \\
& +\left|1-\frac{1}{\gamma}\right| \sum_{j=M+1}^{m-1} \frac{|\gamma|^{j-m}}{\rho^{j}}\left(\rho^{j} \sigma(j)\right),
\end{aligned}
$$

and the monotonicity of $\rho^{m} \sigma(m)$ implies (26) with

$$
K_{2}=1+\frac{1}{|\gamma|}+\frac{\rho|\gamma-1|}{|\gamma|(|\gamma|-\rho)} \text {. }
$$

Lemma 2. The sequence $F_{r}$ defined by (20) for $m \geq M$ is in $\mathscr{B}$.

Proof. We apply Lemma 1 with $w(j)=p_{i}(j)$ and $\gamma=\lambda_{r} / \lambda_{k}$. From (17),

$$
\sum_{j=m}^{\infty} \lambda_{k}^{-j} P_{r}(j)=\sum_{i=1}^{n} \lambda_{r}^{n-i} \sum_{j=m}^{\infty}\left(\frac{\lambda_{r}}{\lambda_{k}}\right)^{j} p_{i}(j) .
$$


Since $\left|\lambda_{r} / \lambda_{k}\right|<1$ for $r<k \leq n$, we can infer from (8) and Lemma 1(a) that

$$
\left|\sum_{j=m}^{\infty} \lambda_{k}^{-j} P_{r}(j)\right| \leq A\left(\frac{\lambda_{r}}{\lambda_{k}}\right)^{m} \phi(m), \quad m \geq M, r \leq k \leq n,
$$

for some constant $A$. Therefore, the second sum in (20) is $O(\phi(m))$. Similarly, Lemma 1 (b) implies that the first sum in (20) is $O(\phi(m))$. Therefore, $F_{r} \in \mathscr{B}$.

In the following lemma let

$$
\zeta(M)=\sup _{m \geq M}[\psi(m) / \phi(m)] .
$$

Since $\psi(m)=o(\phi(m))$ by assumption, $\zeta(M)$ is well defined and

$$
\lim _{M \rightarrow \infty} \zeta(M)=0 .
$$

Lemma 3. If $u \in \mathscr{B}$ then $\mathscr{T}_{r} u \in \mathscr{B}$ and

$$
\left\|\mathscr{T}_{r} u\right\| \leq J \zeta(M)\|u\|,
$$

where $J$ is independent of $u$ and $M$.

Proof. From (18) and (24),

$$
\begin{aligned}
& \left|\sum_{j=m}^{\infty} \lambda_{k}^{-j} \mathscr{M}_{r} u(j)\right| \\
& \quad \leq\|u\| \sum_{i=1}^{n}\left|\lambda_{r}\right|^{n-i} \sum_{j=m}^{\infty}\left|\frac{\lambda_{r}}{\lambda_{k}}\right|^{j}\left|p_{i}(j)\right| \phi(n+j-i), \quad r \leq k \leq n,
\end{aligned}
$$

and

$$
\begin{aligned}
& \left|\sum_{j=M}^{m-1} \lambda_{k}^{-j} \mathscr{M}_{r} u(j)\right| \\
& \quad \leq\|u\| \sum_{i=1}^{n}\left|\lambda_{r}\right|^{n-i} \sum_{j=M}^{m-1}\left|\frac{\lambda_{r}}{\lambda_{k}}\right|^{j}\left|p_{i}(j)\right| \phi(n+j-i), \quad 1 \leq k \leq r-1 .
\end{aligned}
$$

Now (9) and (30) imply that

$$
\left|\sum_{j=m}^{\infty} \lambda_{k}^{-j} \mathscr{M}_{r} u(j)\right| \leq \alpha\|u\|\left|\frac{\lambda_{r}}{\lambda_{k}}\right|^{m} \psi(m), \quad r \leq k \leq n,
$$

where $\alpha$ is independent of $u$ and $M$. By applying Lemma $1(\mathrm{~b})$ with $w(j)=$ $\left|p_{i}(j)\right| \phi(n+j-1)$ and $\gamma=\lambda_{r} / \lambda_{k}$, we see from (31) that

$$
\left|\sum_{j=M}^{m-1} \lambda_{k}^{-j} \mathscr{M}_{r} u(j)\right| \leq \beta\|u\|\left|\frac{\lambda_{r}}{\lambda_{k}}\right|^{m} \psi(m), \quad 1 \leq k \leq r-1,
$$

where $\beta$ is independent of $u$ and $M$. From (23) and the last two inequalities we see that $\left|\mathscr{T}_{r} u(m)\right| \leq J \psi(m)\|u\|(m \geq M)$ for some $J$ independent of $u$ and $m$. This together with (24) and (27) implies (29). 
We can now complete the proof of Theorem 1. Lemmas 2 and 3 and (22) imply that $\mathscr{T}_{r}$ maps $\mathscr{B}$ into itself. If $u_{1}$ and $u_{2}$ are in $\mathscr{B}$, then Lemma 3 implies that

$$
\left\|\mathscr{T}_{r} u_{1}-\mathscr{T}_{r} u_{2}\right\| \leq J \zeta(M)\left\|u_{1}-u_{2}\right\|
$$

Because of (28), we can choose $M$ so large that $\zeta(M)<1 / J$; then the mapping defined by (22) is a contraction mapping of $\mathscr{B}$ and its fixed point $v_{r}$ satisfies (19) for $m \geq M$. Therefore, $y_{r}$ as defined by (21) satisfies (1) for $m \geq M$ and has the asymptotic behavior (11).

\section{A REMARK AND EXAMPLES}

Remark 1. Since $v_{r}$ is the fixed point of (22), we have $v_{r}=F_{r}+\mathscr{T} v_{r}$, where $F_{r}=O(\phi)$ and $\mathscr{T} v_{r}=O(\psi)$. Since $\psi(m)=o(\phi(m))$, the asymptotic formula (11) can be written more precisely as

$$
y_{r}(m)=\lambda_{r}^{m}\left(1+F_{r}(m)+O(\psi(m))\right),
$$

where $F_{r}$, which is $O(\phi(m))$, is the known sequence defined by (20).

In the following examples we consider the difference equation

$$
y(m+2)+\left(a_{1}+\varepsilon(m) / m\right) y(m+1)=a_{2} y(m)=0,
$$

where

$$
\lambda^{2}+a_{1} \lambda+a_{2}=\left(\lambda-\lambda_{1}\right)\left(\lambda-\lambda_{2}\right)
$$

with $0<\left|\lambda_{1}\right|<\left|\lambda_{2}\right|$.

Example 1. Let $\varepsilon(m)=1 / m$. Applying Theorem 2 with $\beta(m)=1 / m^{2}$ and $\gamma(m)=\sum_{j=m}^{\infty} 1 / j^{2}=O(1 / m)$ shows that (32) has solutions $y_{1}$ and $y_{2}$ such that

$$
\begin{aligned}
& y_{1}(m)=\lambda_{1}^{m}(1+O(1 / m)), \\
& y_{2}(m)=\lambda_{2}^{m}(1+O(1 / m)) .
\end{aligned}
$$

However, Theorem 3 and Remark 1 yield sharper estimates

$$
\begin{aligned}
& y_{1}(m)=\lambda_{1}^{m}\left(1+F_{1}(m)+O\left(1 / m^{2}\right)\right), \\
& y_{2}(m)=\lambda_{2}^{m}\left(1+F_{2}(m)+O\left(1 / m^{2}\right)\right),
\end{aligned}
$$

where Lemma 2 implies that the known sequences $F_{1}(m)$ and $F_{2}(m)$ are $O(1 / m)$.

Example 2. Let $\varepsilon(m)=(-1)^{m} / m$. Then Theorem 2 implies only that (32) has solutions satisfying (33). However, now (8) and (9) hold with $\phi(m)=1 / \mathrm{m}^{2}$ and $\psi(m)=1 / \mathrm{m}^{3}$, respectively, so Theorem 3 and Remark 1 yield the sharper estimates

$$
\begin{aligned}
& y_{1}(m)=\lambda_{1}^{m}\left(1+F_{1}(m)+O\left(1 / m^{3}\right)\right), \\
& y_{2}(m)=\lambda_{2}^{m}\left(1+F_{2}(m)+O\left(1 / m^{3}\right)\right),
\end{aligned}
$$

where Lemma 2 implies that $F_{1}(m)$ and $F_{2}(m)$ are $O\left(1 / m^{2}\right)$.

More generally, let $\varepsilon(m)=(-1)^{m} \delta(m)$, where $\delta(m)$ is nonincreasing,

$$
\lim _{m \rightarrow \infty} \delta(m)=0, \quad \text { and } \quad \liminf _{m \rightarrow \infty} \frac{\delta(m+1)}{\delta(m)}>\left|\frac{\lambda_{1}}{\lambda_{2}}\right|^{1 / 2} \text {. }
$$


Then Theorem 3 and Remark 1 imply that (32) has solutions such that

$$
\begin{aligned}
& y_{1}(m)=\lambda_{1}^{m}\left(1+F_{1}(m)+O\left(\delta^{2}(m) / m\right)\right), \\
& y_{2}(m)=\lambda_{2}^{m}\left(1+F_{2}(m)+O\left(\delta^{2}(m) / m\right)\right),
\end{aligned}
$$

where Lemma 2 implies that $F_{1}(m)$ and $F_{2}(m)$ are $O(\delta(m) / m)$. However, Theorem 2 does not apply unless $\sum^{\infty} \delta(j) / j<\infty$, in which case it yields the weaker estimates

$$
\begin{aligned}
& y_{1}(m)=\lambda_{1}^{m}(1+O(\gamma(m))), \\
& y_{2}(m)=\lambda_{2}^{m}(1+O(\gamma(m))),
\end{aligned}
$$

where $\gamma(m)=\sum_{j=m}^{\infty} \delta(j) / j$.

\section{REFERENCES}

1. C. V. Coffman, Asymptotic behavior of solutions of ordinary difference equations, Trans. Amer. Math. Soc. 110 (1964), 22-51.

2. M. Evgrafov, The asymptotic behavior of solutions of difference equations, Dokl. Akad. Nauk SSSR 121 (1958), 26-29. (Russian)

3. A. Gelfand and I. Kubenskaya, On a theorem of Perron in the theory of difference equations, Izv. Akad. Nauk. SSSR Ser. Mat. 17 (1953), 83-86. (Russian)

4. O. Perron, Über Stabilität und asymptotisches Verhalten der Lösungen eines Systemsendlicher Differenzengleichungen, J. Reine Angew. Math. 161 (1929), 41-64.

5. H. Poincaré, Sur les equations linéaires aux différentielles ordinaires et aux différences finies, Amer. J. Math. 7 (1885), 203-258.

Department of Mathematics, Trinity University, 715 Stadium Drive, San Antonio, TEXAS 78212

E-mail address: wtrench@trinity.edu 